\title{
Mini-dose of oxytocin at the beginning of spinal anaesthesia for Caesarean section: the influence on uteroplacental blood flow
}

\section{Fesenko V.1, Nazarenko L.. ${ }^{2}$, Kozmuk O. ${ }^{2}$, Nastenko O. ${ }^{2}$, Torroni P. ${ }^{3}$}

${ }^{1}$ Lviv National Medical University, Dept of

Anaesthesiology \& Intensive Care, Lviv, Ukraine; ${ }^{2}$ Kharkiv City Clinical Maternity Hospital \#6, Dept of Anaesthesiology \& Intensive Care, Kharkiv, Ukraine; ${ }^{3}$ Civitanova Marche Community Hospital, Dept of Anaesthesiology \& Intensive Care, Civitanova Marche, Italy.

Background and Goal of Study: Our previous results [1] showed that in spinal anaesthesia (SA) for Caesarean section (CS), Torroni's technique of i.v. administration of $0.5 \mathrm{IU}$ of oxytocin immediately after SA and before delivery significantly attenuated maternal arterial hypotension (22\% higher minimal systolic blood pressure) and lessened phenylephrine use (2.8 times less dose before delivery, 2.4 times less total dose during surgery). The goal of our study was to assess the possible influence of this technique on the uteroplacental blood flow.

Materials and Methods: SA for elective CS was performed in 12 women, randomly divided into study $(n=6)$ and control $(n=6)$ groups. In the study group, slow i.v. bolus of oxytocin (0.5 IU) was administered immediately after SA performance. Uterine and umbilical circulation blood flow was measured just before and 5-6 min after performance of SA.

Results and Discussion: Right uterine artery systolic/diastolic ratio (SDR) in the study and control groups $(\mathrm{M} \pm \mathrm{SD})$ was, resp., $1.69 \pm 0.09$ and $2.07 \pm 0.68$ before, $3.49 \pm 1.80$ and $3.09 \pm 1.26$ after SA performance, all differences insignificant; resistance index (RI) was, resp., $0.40 \pm 0.03$ and $0.51 \pm 0.12$ before, $0.65 \pm 0.10(p=0.004$ vs pre-anaesthetic) and $0.56 \pm 0.14$ after SA performance. Left uterine SDR was, resp., $1.75 \pm 0.21$ and $2.07 \pm 0.61$ before, $3.77 \pm 1.83$ ( $p=0.041$ vs pre-anaesthetic) and $3.38 \pm 0.95$ ( $p=0.035$ vs pre-anaesthetic) after SA performance; RI was, resp., $0.42 \pm 0.06$ and $0.49 \pm 0.10$ before, $0.68 \pm 0.11$ ( $p=0.003$ vs pre-anaesthetic) and $0.67 \pm 0.09$ ( $p=0.018$ vs pre-anaesthetic) after SA performance. Umbilical arterial SDR was, resp., 2.49 \pm 0.26 and $2.59 \pm 0.31$ before, $2.92 \pm 0.93$ and $2.72 \pm 0.87$ after SA performance; RI was, resp., $0.59 \pm 0.05$ and $0.58 \pm 0.06$ before, $0.62 \pm 0.13$ and $0.59 \pm 0.11$ after SA performance. Any intergroup difference was not significant.

The moderately increased resistance of uterine arteries during SA in both groups may result from both regional analgesia and uterine contractions. Umbilical arterial RI did not change significantly.

Conclusion: Torroni's technique during spinal anaesthesia for Caesarean section has no negative influence on uteroplacental blood flow. right uterine artery systolic/diastolic ratio

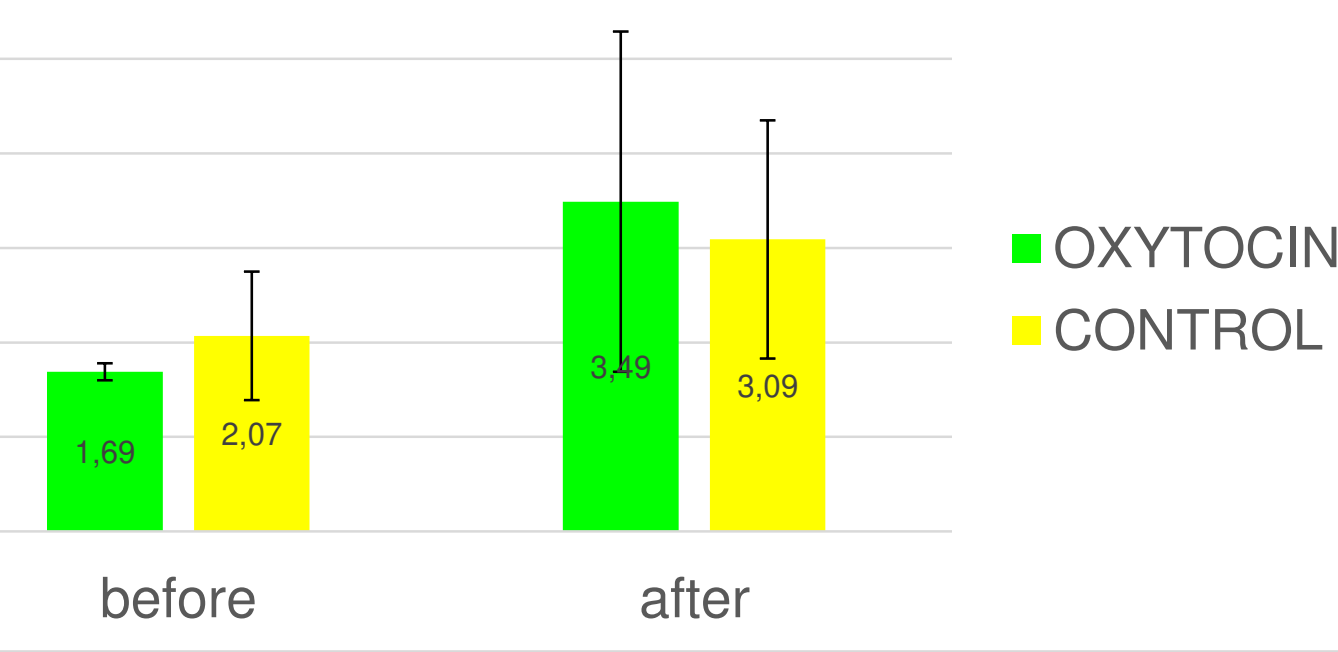

right uterine artery resistance index

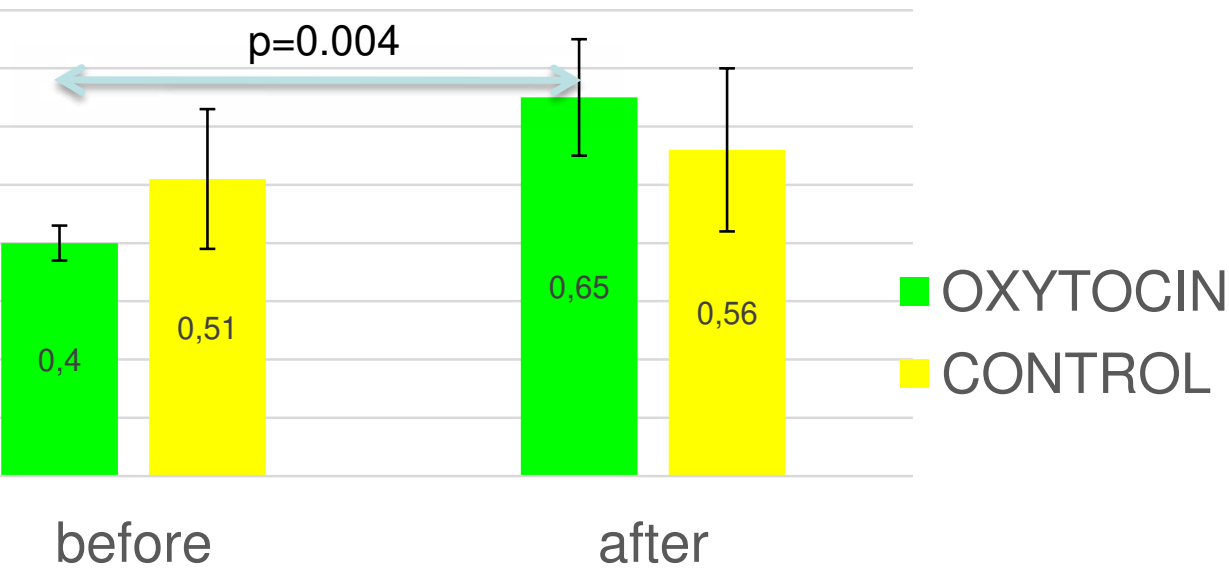

left uterine artery systolic/diastolic ratio

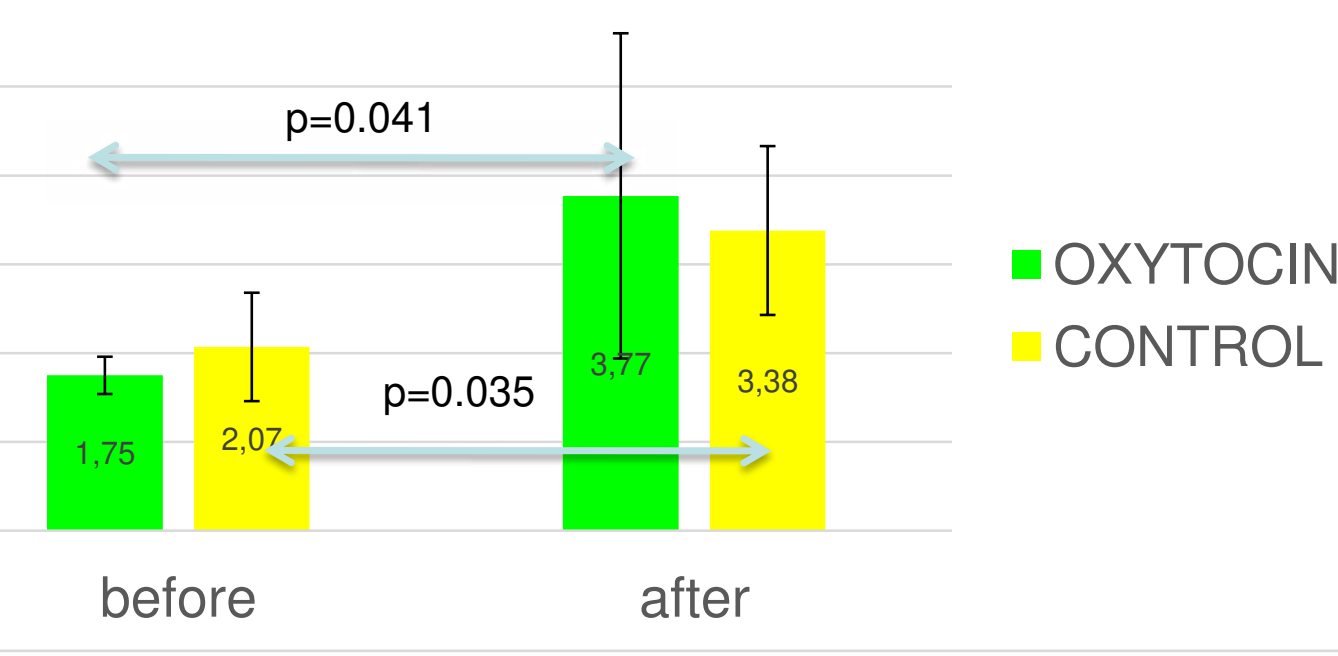

left uterine artery resistance index

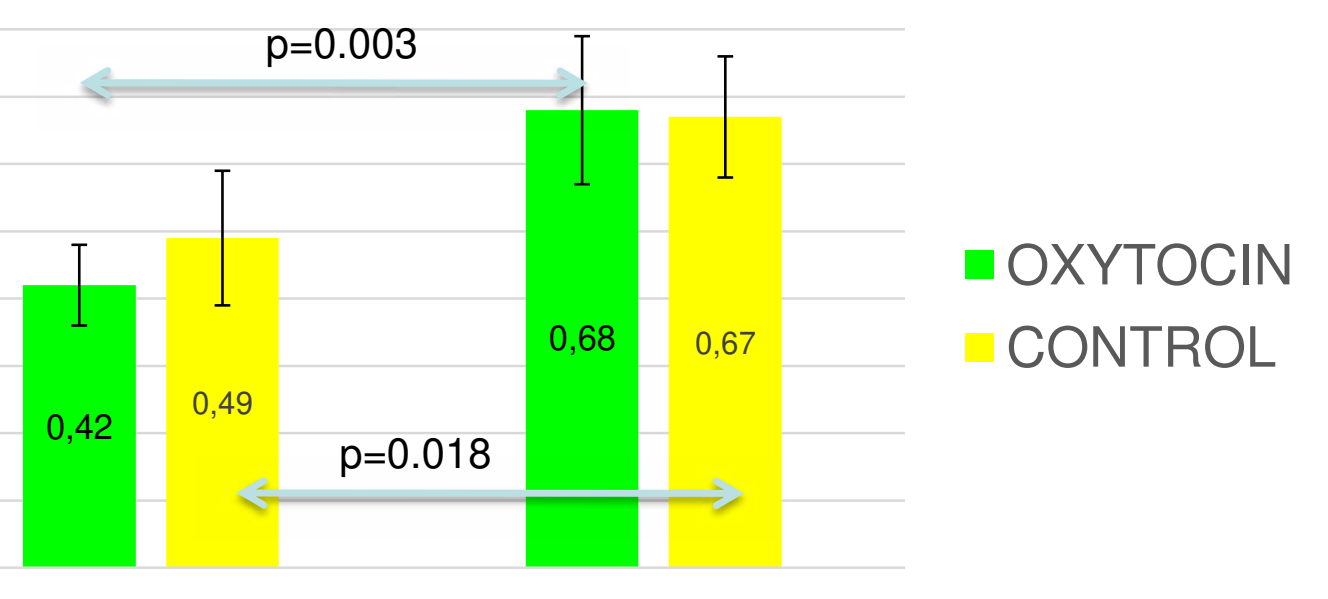

before after

umbilical artery systolic/diastolic ratio

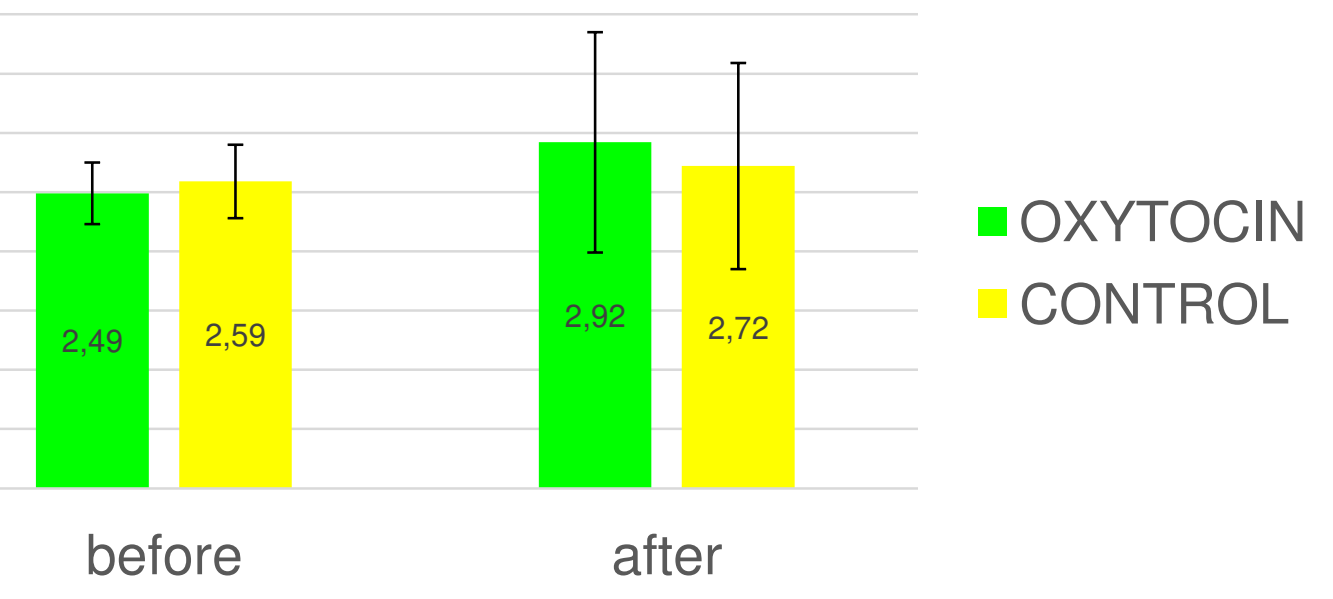

umbilical artery resistance index

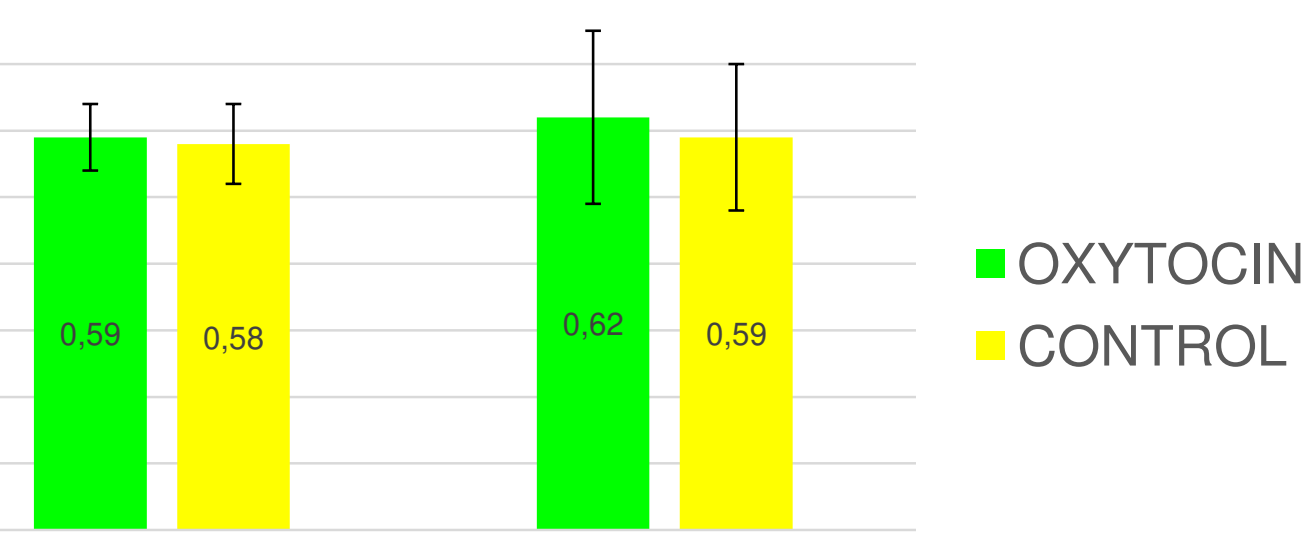

